\title{
Research on the Development Dilemma and Solution Ideas of China's Medicine Logistics Under the Development Trend of Contemporary Logistics
}

\author{
Jiahao $\mathrm{Ge}^{1, *}$, Yang $\mathrm{Gao}^{2}$, Yu Miao ${ }^{1}$, Yanling $\mathrm{He}^{1}$ \\ ${ }^{1}$ School of Economics and Management, Nanjing Institute of Technology, Nanjing, Jiangsu 211100, P.R. China \\ ${ }^{2}$ School of Pharmacy, Nanjing University of Chinese Medicine, Nanjing, Jiangsu 210000, P.R. China \\ *Corresponding author. Email: 2500466475@qq.com
}

\begin{abstract}
As the traditional Chinese medicine industry develops, the logistics of this industry has shown its importance more. On the basis of the analysis of the main problems Chinese medicine logistics faces, such as chaos in the supply market, high transportation costs, backward logistics technology, lack of talents, and lack of dregs disposal, this paper proposes a modernized logistics system based on the special characteristics of Chinese medicine logistics, which includes introducing Chinese medicine information management system, using the scale advantage to ensure the quality of raw materials, adopting new Chinese medicine maintenance technology, realizing standardized packaging, speeding up the training of Chinese medicine logistics talents, and improving the recycling process of dregs, so as to research and explore the goal of establishing a modernized Chinese medicine logistics system of "standardization, informationization, intensification and resourcefulness".
\end{abstract}

Keywords: Chinese herbal medicine, logistics management, pharmaceutical logistics.

\section{OVERVIEW OF CHINESE MEDICINE LOGISTICS}

\subsection{Prospects And Significance Of The Development Of Chinese Medicine}

\subsubsection{Prospects For Chinese Medicine Consumption}

As the Reform and Opening up is deepening, China's development has gained momentum and its economic ties with the other countries have deepened. As one of the calling cards of China's foreign relations, the Chinese medicine industry has gradually formed a global consumer market with a large consumer population and a huge scale.

The rich geographical landscape, diverse environmental climate, abundant land resources and large agricultural population that China owns have created a wide variety of traditional Chinese medicines with diverse medicinal effects. This industrial advantage has made China the world's largest producer of Chinese herbal medicines, with the largest number of varieties and the most complete production system. The concept of "health brings strength" put forward at the 18th National Congress of the CPC and the increasing ageing of the population have made people pay more attention to their health, which has led to the development of the Chinese medicine health industry. As a result, the consumer market for Chinese herbal medicines in China is continuously expanding and consumer habits are gradually deepening. When China's economic development and foreign exchanges become more frequent, the Chinese people's habit of using Chinese medicine continues to radiate to neighbouring countries, and with more international academic exchanges and friendly support, Chinese medicine is gradually moving from China to Asia and the world. 


\subsubsection{Importance Of Chinese Medicine Development}

With regards to clinically, there is tremendous space for exploration in the study of Chinese medicine. There is a wide variety of Chinese medicines, and with the perfection of purification and separation techniques, the huge amount of data brought about by pharmacological experiments on Chinese medicines has provided new directions for the treatment of existing diseases. In terms of practicality, China has a long history of cultivation, breeding, research and use of Chinese herbal medicines, with a deep popular heritage. The experience of the development and use of Chinese herbal medicine shows that it can play a role in the prevention and treatment of disease. This is the reason for the establishment of traditional Chinese medicine departments in hospitals and the growth in consumption of traditional Chinese medicine. Through the upgrading of production processes, herbal preparations have evolved step by step from traditional dosage forms such as tonics, poultices, elixirs, pills, dispersants and liquors to new dosage forms such as tablets, capsules, aerosols and injections, which contain multiple active ingredients that show better efficacy than single active compounds and in some cases can exhibit therapeutic effects that are not possible with single compounds and have milder and longer lasting effects with lower toxicity [1].

Turning to the economic aspect, China's herbal medicine cultivation bases are mainly located in less developed regions such as the northeast, central, western and southern regions. The production and distribution of Chinese herbal medicines can boost the local economy and economic ties with developed regions. At the same time, as the world's largest exporter of Chinese herbal medicines, China's growing overseas demand provides net exports to customs that can generate a large trade surplus for the country.

\subsection{Overview Of The Main Logistics Segments}

\subsubsection{Overview Of The Transport Chain}

In terms of logistics and transportation, Chinese herbal medicines still retain their original attributes as agricultural and by-products after primary processing at origin. However, after processing into manufactured products, the commodity attributes of Chinese herbal medicines change from agricultural products to pharmaceutical products. Although both need to comply with the Code of Practice for the Production and Quality Management of Chinese Herbal Medicinal Materials (GAP), as far as the logistics link is concerned, because of the different commodity attributes, Chinese herbal medicine logistics are roughly divided into: 1, upstream raw material agricultural logistics; 2, downstream manufactured product pharmaceutical logistics (see Figure 1).

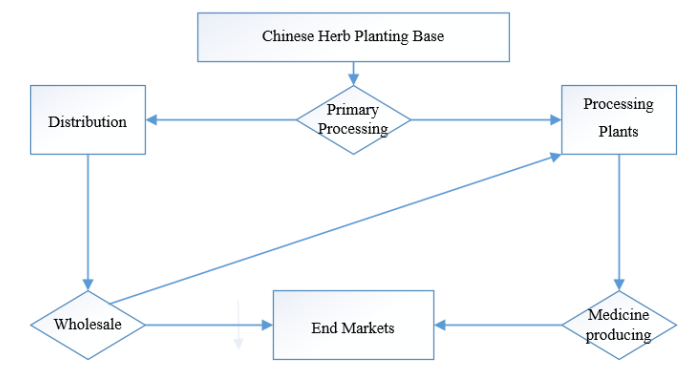

Figure 1 Chinese medicine transport flow

In raw material logistics, the total annual logistics of Chinese herbal medicines in China is about 17 million tons, with bulk Chinese herbal medicines and fine precious and poisonous hemp-restricted Chinese herbal medicines accounting for more than $80 \%$ [2]. Affected by factors such as distribution of origin, road conditions, preliminary processing links, logistics facilities and storage conditions, logistics specialization is highly demanding and intermediate links must be coherent and standardized to ensure raw material quality. It is specifically proposed that, in terms of distribution and processing, unlike ordinary agricultural products, downstream links have different requirements for the freshness of Chinese herbs and to ensure that Chinese herbs are not contaminated from the beginning of the acquisition process, production sites need to be equipped with corresponding distribution and processing sites and facilities and equipment. In the logistics of manufactured products, the products of Chinese herbal medicine are mainly divided into Chinese herbal medicine tablets, health products, Chinese patent medicines and Chinese herbal medicine extracts. The sales channels of Chinese herbal medicines are mainly through the several major distribution centers of Chinese herbal medicines across the country to the end of pharmacies, pharmaceutical companies, hospitals and other consumer objects for the wholesale and retail of Chinese herbal medicines, where pharmaceutical companies also need to carry out further production and processing before transporting to the end market. In the logistics chain, the requirements in terms of temperature control, humidity control and maintenance technology are also more stringent in order to avoid contamination and destruction of Chinese herbs. Therefore, the construction of downstream logistics needs to rely on the development of cold chain logistics and new conservation technologies in order to achieve a deeper and more distant market.

\subsubsection{Overview Of the Storage Chain}

Unlike general goods, in terms of special storage and transport requirements, Chinese herbs employ a unique 
method of storage. Herbal storage requires a certain degree of specialization, faced with a wide variety of herbal medicines. Factors affecting the variability of Chinese herbal medicines include subjective factors such as chemical composition and its nature and water content, and include objective factors such as environment, biology and time. At present, the majority of Chinese herbal medicines in China still use traditional conservation methods, say, dry conservation methods, cold storage conservation methods, buried storage conservation methods, alcohol smothering conservation methods, regular tray mixing methods and chemical conservation methods. The special Chinese herbs need special care: salt-pickled Chinese herbs can be sealed in tanks or altars and stored in cool warehouses below $30^{\circ} \mathrm{C}$; flammable Chinese herbs are prone to oxidation and should be stored separately or in dangerous goods warehouses; fresh Chinese herbs must be controlled with appropriate dryness and humidity, not too dry or too damp to prevent drying out and decay; poisonous and hemp Chinese herbs can be selected for care by sealing, moisture absorption, air conditioning and low temperature methods according to different sources; fine and expensive Chinese herbs should be sealed first and then stored. Fine and precious Chinese medicines should be stored in a dry and cool place after being sealed first [3].

\section{PROBLEMS IN CHINESE MEDICINE LOGISTICS}

\subsection{Chaos In The Chinese Herbal Medicine Supply Market}

The raw materials of Chinese medicine are divided into animal, plant and mineral. Animal and mineral raw materials generally have few varieties, small but stable origins and yields, and relatively low dosage and demand. The quantity and quality of plant herbs are uncertain due to the influence of climate, topography, land and cultivation techniques. Secondly, due to the lack of standards for distribution and market demand guidance, cultivation in inauthentic production areas is widespread, and the herbs circulating to the market do not meet the norms for efficacy, quality and hygiene conditions, and there is no way to trace the production process. Such poor quality herbs not only affect the quality of downstream products, but may also degrade the efficacy of the cultivated herbs. The supply of Chinese herbal medicines is not fully guaranteed, and it greatly restricts the construction of a complete logistics supply chain for Chinese medicines.

\subsection{High Costs For Chinese Medicine Transportation}

At this moment, the costs arising from the logistics link of Chinese herbal medicines are the main component of the total cost, and the causes of high logistics costs at all stages are very complex. From the source, $80 \%$ of Chinese herbal medicines are currently concentrated in the hands of scattered farmers, and the production areas of Chinese herbal medicines are mostly located in the suburbs of the west, northeast and south, where the road conditions are complicated and the traffic order is chaotic, and the transport distance from the main eastern markets and overseas markets spans a large distance; the production of Chinese medicine enterprises needs to be carried out in strict accordance with the national GAP norms, and in their production system and logistics system, whether it is raw materials, both raw materials and finished medicines produced must be traceable, which requires the logistics system of traditional Chinese medicine enterprises to have traceability functions [4]. This, coupled with the unique conservation aspects of Chinese medicine logistics, makes the initial construction and circulation operation costs of Chinese medicine logistics high; the terminals of Chinese medicine sales are generally hospitals, pharmacies and communities, with more dispersed sales outlets, which requires the optimization of end-of-pipe logistics. The complexity of the requirements and the stringency of the requirements make the Chinese medicine logistics system face higher capital investment and operating costs at the beginning of its establishment.

\subsection{Backward Logistics Technology}

The main areas where logistics technology is lagging are as follows:

1. China has more than 12,800 kinds of traditional Chinese medicine (traditional Chinese medicine) resources, which are distributed in a wide range of regions and are extremely territorial, with a wide variety of species and different functions and forms [5]. This is an advantage for the development of the industry, but the complex and huge database poses a great challenge for the information management system of Chinese medicine logistics.

2. Traditional conservation methods are outdated and can easily cause changes in the quality of Chinese herbal medicines or expose them to contamination by pharmaceutical agents, exacerbating the loss and deterioration of the active ingredients of Chinese herbal medicines during transportation and storage, and affecting processing safety.

3. Irregularities in the distribution chain and imperfect packaging standards and commodity standards. For example, the driver in cold chain transportation turns off the refrigeration unit midway to save fuel or the lack of refrigeration in the intermediate links, etc., all of which will lose herbs in the transportation and storage process.

Because of the poor facilities and technology, the biologically active ingredients in Chinese medicine are 
heavily broken in the intermediate stages, resulting in a large amount of losses and waste dregs. The cumulative loss rate at every level and the backwardness of the processing technology make the unit cost of Chinese medicine too high and reduce the profit margin.

\subsection{Lack of Talent}

Unlike ordinary agricultural products, Chinese herbs have a higher added value, and the way they are grown requires a high degree of local intensification to ensure the purity of the active ingredients during the growing process. The logistics of Chinese herbal medicine is therefore highly professional and coherent, and practitioners need to have a certain level of expertise from the planting stage to reduce logistics costs and increase the value of the industry. The current situation is that the corresponding supporting facilities are not sufficiently invested, operator training is lacking and there is a shortage of technical personnel at all levels.

\subsection{Lack Of Recycling Treatment For Medicine Dregs}

China is a major producer of Chinese medicine, but also a major consumer of Chinese medicine resources. The production, processing, preparation and use of Chinese medicine may produce Chinese medicine dregs. Therefore, the amount of Chinese medicine dregs produced in China is huge, with annual emissions reaching 30 million tonnes. The current disposal methods of Chinese medicine dregs are mainly piling, landfill and incineration, of which piling accounts for a large proportion. This simple and crude way of disposal will not only have an impact on the environment, but is also a waste of resources.

\section{IDEAS FOR CHINESE MEDICINE LOGISTICS SYSTEM CONSTRUCTION}

The logistics of Chinese herbal medicines in circulation includes activities like packaging, transportation, warehousing and storage maintenance, circulation processing and information processing of Chinese herbal medicines. Due to the varying quality of Chinese herbal medicines on the market, the lack of standards in the current packaging methods and specifications, the lack of information on packaging markings, and the backward level of warehousing and storage maintenance, secondary contamination such as adulteration and forgery occurs, leading to damage to the quality of Chinese herbal medicines, loss of quality and low level of quality tracing system[6]. The emergence of new logistics technology has broken the original constraints in the field of Chinese medicine logistics process technology [7]. The expansion and upgrade of the new generation of information and communication industry based on $5 \mathrm{G}$ is the key helper to drive the integration and improvement of the information management system of Chinese medicine logistics. As a result of the epidemic, public attention to the field of medicine has increased, and the strong response from the government and the important role played by traditional Chinese medicine in the early stages of the fight against the epidemic have led to a greater awareness of the effectiveness of traditional Chinese medicine. With the support of policies and the inflow of funds relying on the foundation of several existing Chinese herbal medicine distribution centers, a distribution and processing centre was established to improve the overall efficiency of logistics and distribution and complete the entire supply chain. (See Figure 2)

\subsection{Information Management System For Chinese Medicine Logistics}

With the investment in 5G technology and new infrastructure, the national industrial layout is pushing downwards and deeper, and the resistance to the construction of the lower level of Chinese medicine logistics has become less. The third-party traditional Chinese medicine logistics companies have perfected their handling of logistics links after many years of practical experience. In addition, the national standardization system for Chinese medicine has been improved and the commodity codes have been gradually unified. The key supplier management module and commodity management module in the traditional Chinese medicine logistics management system can be greatly improved with the support of technology, experience and policies. By solving the existing problems of information processing capacity in Chinese medicine logistics, the development needs of such links as intensive cultivation, standardized transport, and efficient production can be met.

\subsection{Construction Of Modern Chinese Medicine Logistics System}

\subsubsection{Industry Standards Improvement}

Through the establishment of a standard system for Chinese herbal medicines, information on market prices, circulation quality, commodity codes, production traceability, etc. will be standardized and information management on Chinese medicine logistics will be uploaded. On the one hand, openness and transparency will facilitate the establishment of a standardized system for the industry, and on the other hand, information transfer will improve the efficiency of production and circulation.

\subsubsection{Management System Innovation}

Through the introduction of intelligent logistics technology and logistics information technology [5], the 
distribution and processing process is standardized to reduce errors in the transport and storage process, improve processing speed and capacity, reduce losses caused by improper operation, reduce the costs incurred by intermediate logistics links, and shorten logistics time.

\subsubsection{Central Node Establishment}

By establishing logistics centre nodes in pre-existing large distribution centers such as Bozhou in Anhui, Anguo in Hebei, Zhangshu in Jiangxi and Yuzhou in Henan[8]. Distribution processing treatment is used for Chinese herbal medicines to further ensure the quality and distribution efficiency of Chinese herbal medicines in the storage and transportation process.

\subsubsection{Security Of raw Materials Supply}

Through the agglomeration effect of the authentic production area scale, the original scattered and varying quality of planting scattered households are collected and gradually formed into a large regional planting base, using the advantages of scale to ensure the supply of raw materials.

\subsubsection{Storage And Transportation Technology Upgrade}

Through the use of new herbal storage and maintenance techniques, such as anisotropic anthelmintic conservation, CA storage, far-infrared heating conservation, microwave heating conservation, air curtain moisture conservation, de-oxygenation and freshness conservation and biopesticide control, conservation methods are used. The improved level of conservation technology not only extends the shelf life of herbs and their manufactured products, improves the quality of herbs when they reach the market, but also increases the depth of the market as far as special needs such as fresh herbs are concerned [9].

\subsubsection{Distribution And Processing Specifications}

The standardized packaging of Chinese herbal medicines facilitates the quality assurance of Chinese herbal medicines, not only for storage and transportation, but also for counting and measuring, increasing the added value of Chinese herbal medicines and reducing the loss of Chinese herbal medicines. Having different packaging methods for Chinese herbal medicines, proprietary Chinese medicines and authentic Chinese herbal medicines is also convenient to trace the entire production chain of Chinese herbal medicines.

\subsubsection{Information System Construction}

The traditional Chinese medicine logistics information management system platform provides integrated planning for the production areas, distribution links and sales markets of traditional Chinese medicine, enabling fine handling of operations, coherent linkage and close connection between the upstream and downstream industry chains, helping manufacturers to formulate production and sales plans and broaden sales channels with high-speed information transfer.

Only when the commercial, logistic, information and

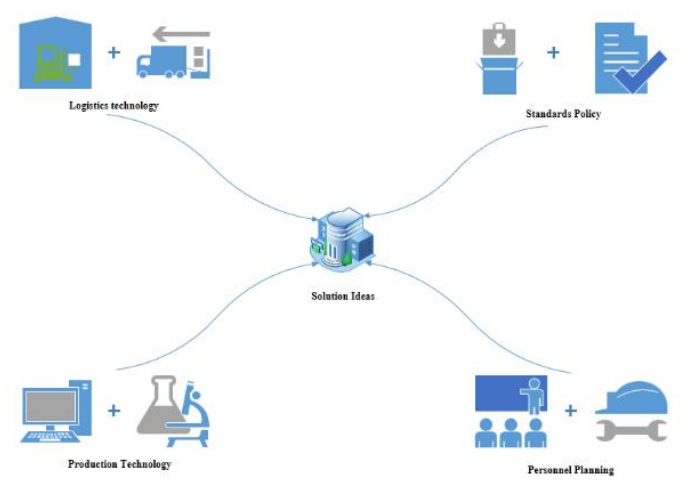

capital flows in upstream and downstream of the Chinese medicine industry chain operate at a higher speed can a modern logistics system with standardization, information technology and intensification be gradually established.

Figure 2 Construction scenario

\subsection{Talent Cultivation Program Construction}

There are two main reasons for the lack of talent in Chinese medicine logistics: 1 , the distribution of Chinese medicine production areas is remote from developed areas, the local grassroots practitioners are poorly educated, and the relevant personnel are not formally trained, unskilled in operation and lack of knowledge of Chinese medicine and logistics skills; 2, influenced by the distribution of production areas, the technical personnel recruited at the current salary level are unable to meet the higher requirements of Chinese medicine logistics in terms of quality and quantity. In this regard, while integrating plantation areas, establishing logistics systems and processing parks, it is important to start from the actual sector and purposefully combine the correct experience of the past with advanced management knowledge, to thereby train and make up the knowledge of Chinese medicine and logistics for the grassroots practitioners. In terms of talent introduction, Chinese medicine logistics technicians need not only a certain knowledge of Chinese medicine, but also relevant logistics knowledge. The existing talent gap and the new gap in the subsequent development process require not only the adjustment of the existing salary system and 
training system by the enterprises, but also the support and subsidies from the policy to the less developed areas.

\subsection{Construction Of The Recycling Chain Of Medicine Dregs}

The entire Chinese medicine production process produces a large amount of dregs, which are traditionally disposed as waste. In fact, the dregs still contain proteins, cellulose, amino acids, trace elements, alkaloids, polysaccharides, flavonoids and other bioactive components. It can be turned into a treasure through composting, cultivating edible mushrooms, making animal feed and producing biogas. Meanwhile, the resourcefulness, minimization and harmless treatment of Chinese medicine dregs is a perfect way to achieve the objectives of the sustainable development strategy.

\section{CONCLUSION}

Although China is rich in Chinese herbal resources, the low level of utilization of Chinese herbal resources has caused high costs and difficulties in ensuring the quality of Chinese herbal materials in the logistics chain, and that has seriously limited the development of the Chinese herbal industry. To solve the problems mentioned above, this paper conducts a preliminary analysis of the problems faced by the existing logistics system of Chinese herbal medicines and proposes preliminary solutions to the problems of market chaos, information circulation difficulties, backward conservation technology, lack of talents, and disposal of dregs, so as to increase the overall level of efficiency of the Chinese herbal medicine industry.

\section{REFERENCES}

[1] Jianping Zhou, Xing Tang. Industrial Pharmacology [M]. Beijing:People's Health Publishing House, 2014

[2] Guidance on Accelerating the Construction of Modern Logistics System for Chinese Herbal Medicines [N]. China Chinese Medicine News,2015-01-08(005).

[3] Liang Xu. Conservation of Chinese medicine [M]. Beijing: Science Press, 2006.1

[4] Chongbin Xi. How to Make a Good Logistics System Project in Chinese Medicine Industry-Interview with Zhang Huimin, Deputy General Manager of Guangdong Xinbao Pharmaceutical Technology Co. Logistics Technology and Application, 2020, 25(10):136-138.

[5] Xingguo Zhang, Tingting Zhu, Kunlun Li. Reflections on the Construction of Modern Chinese
Herbal Medicine Logistics System in China[J]. China Business Theory, 2018(16):13-15.

[6] Hao Xiong Yang, Dan Kong. Combing Quality and Safety Control Policies for Chinese Herbal Medicines in Circulation[J]. China Chinese Medicine Letter, 2017, 24(07):7-10

[7] Feng Xue, Jianyu Huang, Hao Wu, Junsong Li, Liuqing Di, Hongzhi Qiao. Research Progress of Modern Chinese Medicine Processing Technology[J]. Journal of Nanjing University of Traditional Chinese Medicine, 2020, 36(05):727735 .

[8] Fengmao Li. Study on Bottlenecks and Countermeasures for the Development of Chinese Medicine Industry in Bozhou [D]. Supervisor: Youhui Wang. Huazhong Normal University,2018.

[9] Xuewen Zeng, Xiaoqin Hu.The Construction Strategy of Fresh Chinese Herbal Medicine Logistics System Based on the Fresh Use of Chinese Medicine[J]. Modern Business, 2015(14):27-28. 